\title{
UN MYRIAPODE CHILOPODE, ORPHNAEUS BRASILIANUS \\ NIGROPICTUS ATT. DE L'OREILLE HUMAINE \\ EN ÉQUATEUR
}

\section{Par Jean-Marie DEMANGE}

Notre collègue R.-Ph. Dollfus nous ayant donné à déterminer trois Geophilomorphes $\&$, provenant de Puerto Napo et de Pichincha (Equateur), récoltés par le $\mathrm{D}^{\mathrm{r}}$ Luis $\mathrm{A}$. Leon, nous avons reconnu qu'il s'agissait d'Orphnæus brasilianus nigropictus Attems.

Ces Myriapodes furent trouvés vivants dans le conduit auditif externe d'oreilles d'indigènes. Leur présence déterminait une forte douleur et une inflammation de l'oreille externe. Ces symptômes, tout au moins l'inflammation, peuvent s'expliquer par l'irritation due à la sécrétion des pores ventraux.

Le fait que l'on ait trouvé des Myriapodes dans des oreilles humaines est extrêmement intéressant, car ce cas est rare ; il n'a été signalé qu'une ou deux fois, notamment par le $\mathrm{D}^{\mathrm{r}}$ Rouffiandis en 1903 , sous forme de " recette » " pour enlever un petit millepattes qui a pénétré dans l'oreille ». De plus, l'espèce qui nous occupe n'a pas encore été signalée dans l'oreille (1), ni dans les fosses nasales, ni dans le tube digestif d'êtres humains.

Les descriptions de ce Myriapode publiées jusqu'ici sont incomplètes et les figures peu nombreuses. Il est donc intéressant de les illustrer et de les compléter :

Longueur, 55 à $70 \mathrm{~mm}$.

Segments pédifères, 79 ( $($ ).

Corps robuste et long, un peu atténué en avant. Tête plus étroite que le tronc, plus large que longue. Antennes courtes, fortement pileuses, très rapprochées à la base et épaisses, de 14 articles.

Labre (fig. 1) à bord légèrement incurvé, avec un léger renflement à la partie médiane, planté de fines denticulations.

(1) D'ailleurs, les Myriapodes que l'on a pu trouver dans le conduit auditif externe sont encore inconnus.

Ann. de Parasitologie, t. XXII, nos $1-2,1947$, p. 65-67.

5. 
Mandibules avec cinq peignes, à dents fortes et écartées. Les premiers peignes composés de 26 à 27 dents environ.

Clypeus parsemé de nombreuses soies rassemblées en un champ, dont le grand axe est perpendiculaire à l'axe du corps.

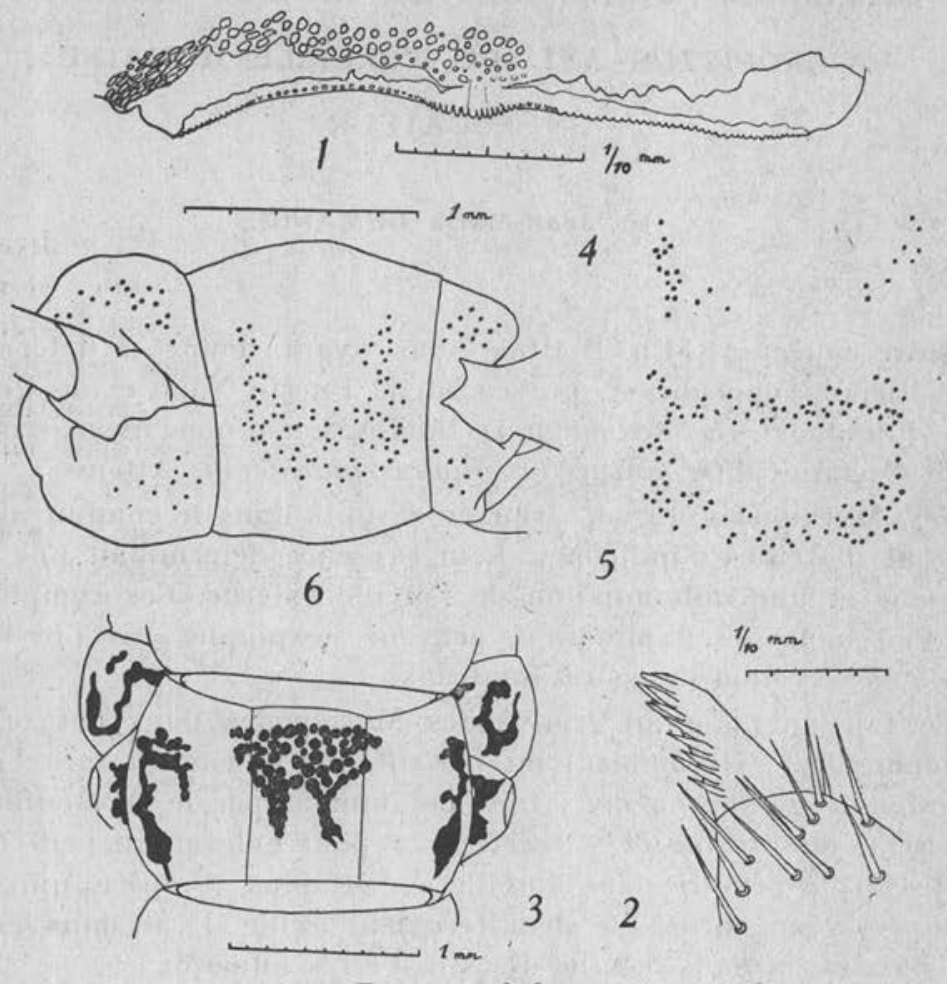

Fig. 1. - Labre

Fig. 2. - Griffe apicale droite du télopodite des deuxièmes inâchoires

Fig. 3. - Tergite

Fig. 4. - Champs poreux du premier sternite

Fig. 5. - Champ poreux du deuxième sternite

Fig. 6, - Champ poreux du pénultième sternite

Bord externe du télopodite des premières mâchoires portant deux palpes. Bord rostral du coxosternum des deuxièmes mâchoires sans sillon médian. Ongle apical excavé et pointu, les bords portant de nombreuses épines (fig. 2).

Coxosternum forcipulaire large; le bord antérieur faiblement échancré, lignes chitineuses complètes. Forcipules courtes ne dépassant pas le bord antérieur de la tête. Tergite forcipulaire court et très large, de forme trapézoïdale. 
Tergites à surface inégale, parcourus par deux sillons longitudinaux profonds, déterminant une bande médiane saillante. Les paratergites font leur apparition successivement d'avant en arrière. On compte un paratergite intercalaire et un paratergite principal. Tergites et pleurites stigmatifère et intercalaire tachés de noir; les tergites ont une tache, au milieu, envoyant des prolongements en arrière et flanquée de deux autres masses noires (fig. 3 ). Les taches ne se trouvent que dans la région antérieure du corps, disparaissant progressivement dans la partie postérieure.

Stigmate ovale.

Pores ventraux condensés en des champs de formes diverses d'avant en arrière, Présents à tous les sternites. Premier sternite possédant des masses latérales de pores, dont les parties postérieures tendent à se rapprocher (fig. 4). Pores du deuxième sternite disposés en une bande continue suivant les contours du sternite (fig. 5). Dans le milieu du corps jusqu'à la fin, pores rassemblés en un champ ayant la forme d'un U, dont la partie postérieure arrondie est très épaissie. Pores ventraux également présents sur le procoxa et le métacoxa (fig. 6). Tergite du dernier segment pédifère large et court, au bord postérieur faiblement arrondi.

Hanches des pattes terminales larges et courtes et entièrement dépourvues de pores.

Corps entier de l'animal ponctué, très fortement dans la partie antérieure (tête, coxosternum forcipulaire, forcipules), plus faiblement dans le milieu.

\section{Bibliographie}

Brumpt (E.). - Précis de parasitologie, t. II. Collection Précis médicaux, Paris, $5^{\circ}$ éd., 1936.

Neveu-Lemaine (M.). - Traité d'entomologie médicale et vétérinaire, Paris, Vigot, 1938.

Rouffiandis (V.). - Géographie médicale. Le Moyen Laos. Ann, Hyg. méd, coloniales, VI, 1903.

Laboratoire de zoologie du Muséum nat. d'Hist. nat., Paris 